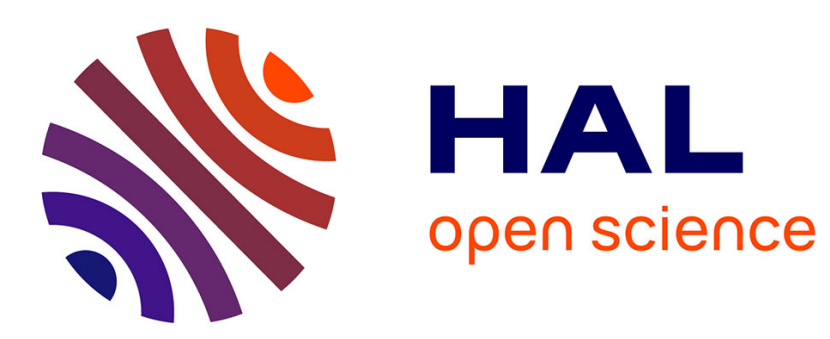

\title{
Extreme value theory for the study of probabilistic worst case delays in wireless networks
}

\author{
Alexandre Mouradian
}

\section{To cite this version:}

Alexandre Mouradian. Extreme value theory for the study of probabilistic worst case delays in wireless networks. Ad Hoc Networks, 2016, 48, pp.1 - 15. 10.1016/j.adhoc.2016.05.006 . hal-01398533

\section{HAL Id: hal-01398533 \\ https://hal.science/hal-01398533}

Submitted on 17 Nov 2016

HAL is a multi-disciplinary open access archive for the deposit and dissemination of scientific research documents, whether they are published or not. The documents may come from teaching and research institutions in France or abroad, or from public or private research centers.
L'archive ouverte pluridisciplinaire HAL, est destinée au dépôt et à la diffusion de documents scientifiques de niveau recherche, publiés ou non, émanant des établissements d'enseignement et de recherche français ou étrangers, des laboratoires publics ou privés. 


\title{
Extreme Value Theory for the Study of Probabilistic Worst Case Delays in Wireless Networks
}

\author{
Alexandre Mouradian \\ Laboratoire des Signaux et Systèmes (L2S, UMR8506), Univ. Paris \\ Sud-CNRS-CentraleSupélec, Université Paris-Saclay F-91192 Gif-sur-Yvette \\ alexandre.mouradian@u-psud.fr
}

\begin{abstract}
Wireless networks are more and more envisioned to be used as a support for critical safety applications. It is notably the case for large scale wireless networks such as vehicular networks, for which safety is one of the main motivations for their development. In this context, the system designer must be able to predict bounds on Quality of Service (QoS) parameters such as delay and delivery ratio. Nevertheless, obtaining strict bounds on such parameters is often difficult because of the unpredictability of the environment (electromagnetic interference, user mobility, etc). Even when the environment is well characterized, the derivation of the bound might be impractical because of the complexity of the models and techniques (the combinatorial explosion problem of model checking is an example) or the bound derived might not be tight (for example with Network Calculus). On the other hand, classic network performance evaluation techniques (stochastic modeling, discrete event simulation, experimentation, etc) usually focus on parameter averages and give very few insights on the extreme deviations from these averages which are of paramount importance for critical applications.

In this paper, we propose to use the Extreme Value Theory (EVT) in order to investigate worst case delays in wireless networks. EVT is a statistical tool which allows to make predictions on extreme deviations from the average. These statistical predictions can be made based on data gathered from simulation or experimentation. We first briefly introduce the technique. Then we discuss its application to the study of delays in wireless networks and we illustrate our discussion with a case study: safety applications in vehicular networks.
\end{abstract}

Keywords: Extreme Value Theory, Worst-Case delays, Large-Scale 
Wireless Networks, Real-Time

\section{Introduction}

Real time critical applications are applications which have strong timing requirements. If they miss a deadline it can have dramatic consequences on the environment or even on human lives. More and more of these critical applications are now distributed and thus rely on networks. One example, that will be considered through this paper, is safety applications for connected cars. These applications are one of the main motivation for the deployment of Vehicular Adhoc NETworks (VANETs) [1] for cars. These road safety applications mainly aim at providing an assistance to the driver in order to avoid car accidents and are described in more details in section 5.1. Such critical applications have strong timing requirements. It is thus of paramount importance to design network protocols which can provide bounded worst case delays. But more importantly, the bounds have to be known and have to comply with the requirements of the target application.

Unfortunately, most of the performance evaluation techniques of the literature such as stochastic models, simulation and experimentation focus on parameter averages [2] and give very few insights on extreme cases. To address this issue, formal methods have been developed specifically to study real-time critical systems [3] [4]. These methods aim at deriving bounds on the response time of the system and prove that the system will never miss a deadline. Nonetheless, many problems may arise when using these methods. First, it is often difficult to define and predict the behavior of the system because it may depend on complex probabilistic environmental events. For example, a packet may be lost because of unexpected electromagnetic interferences or mobility. Most of the classic formal methods of the literature do not allow to model efficiently this kind of events [3] [4]. Even when it is possible to handle such probabilistic events, formal methods allow to prove properties only on a model of the system. The level of details of the model is often limited because if it is too complex, the use of formal methods can become impractical or even impossible. It is the case, for example, for the model checking [3] technique whose combinatorial explosion is a well known limitation (this issue is evoked in Section 2).

In this paper, we propose to estimate the probabilistic worst case delay in a network using statistical tools. The probabilistic worst case delay is 
defined as the probability that the maximum delay is less than a given value (the delay can be a packet end-to-end delay, an inter packet delay, a one-hop delay, etc.). The main advantage of the statistical approach is that it allows to evaluate directly the real studied system (or a highly detailed simulation model) instead of working on an abstracted theoretical model. Moreover, the obtained probabilistic worst case delay has good chances to be less pessimistic than the worst case derived using classic formal methods as it is the case for worst case execution times in the literature [5].

The statistical method used in this paper is Extreme Value Theory (EVT) [6]. It is presented in details in sections 3 and 4 . The method can be summed up in the three following steps:

1. Produce/Gather data (by simulation or experimentation).

2. Extract extreme values from the data (maxima or values over a threshold).

3. Fit extracted values to a probability distribution predicted by the theory.

In this paper, in order to demonstrate the effectiveness of the method in the context of probabilistic worst case delay estimation in networks, we apply EVT to the case of VANETs safety applications. This choice is motivated by the fact that classic formal methods have difficulties to handle such networks because of the scale and the high mobility of the network and the error-prone wireless medium. We gather the data from extensive simulation of a large scale realistic urban vehicular network.

The key contributions of this paper can be summed up as follows. This work represents, up to our knowledge, the first attempt to apply EVT to the study of probabilistic worst case delays in wireless networks. Moreover, we simulate the vehicular network safety applications using a realistic large scale mobility trace [7]. This paper is with [8] one of the first evaluation of vehicular critical applications using the 802.11p [9] standard in a realistic large scale urban environment ([8] focuses on the probability of delivery, and we focus on the probabilistic worst case delay).

We can notice that even if we consider VANETs safety applications in this paper, the method and discussions we develop are in principle applicable to any computer network.

This paper is an extension our previous work [10] and explore in more details the EVT techniques and its applicability to the study of delays wireless networks. We notably add the description and the application of a second 
theorem of EVT and we study the parametrization of the method, whereas [10] is more focused on the application.

The remainder of the paper is organized as follows. In section 2 , the related work is presented and commented. In section 3, we introduce EVT's main theorems. Section 4 consists in a discussion on the application of EVT to the study of large delays in wireless networks. In section 5, we introduce safety applications for VANETs, we present the simulation setup and we apply EVT to study the delays of vehicular networks safety applications. Section 6 provides the conclusion remarks and lists future works.

\section{Related work}

The scientific literature concerning network performance analysis focuses mostly on parameter averages [2]. Indeed, performance evaluation techniques such as theoretical modeling (notably stochastic models), simulation and experimentation mainly deal with throughput, loss rate (or delivery ratio) and delay averages metrics. Nevertheless, for critical applications, averages are not sufficient [11]. In the case of critical real-time applications, if a packet delay deviates from the average and misses a deadline, the consequences on the environment or even on human lives can be catastrophic [11]. To deal with this issue, over the last decades, the real-time systems research community has developed methods and models to formally derive worst case delays and formally prove that a system respects timing constraints.

The main formal methods used to check timing behaviors of systems and derive the worst case response time are theorem proving and model checking [12]. Theorem proving consists in expressing the system behavior as mathematical formulas and proving that the system satisfies a desired set of properties. Process algebras [13], for instance, fall into this category. In the case of computer networks, Network Calculus [4] provides a powerful formalism to derive worst case end-to-end delays. Nevertheless, it suffers from some limitations: the derived bound can be pessimistic which leads to design oversized systems. Moreover, Network Calculus is not adapted to dynamic networks: it cannot easily handle load balancing or mobility for instance [4].

On the other hand, timed model checking proposes a method to automatically and exhaustively explore all the behaviors (executions) of the modeled system in order to assess that its timing behavior is correct (that it respects a set of properties) [3]. As this method explores all the possible behaviors of 
the system, the exact worst case delay can be found. Nevertheless, the main drawback of this method is what is called the combinatorial explosion problem: the number of possible behaviors of the system increases exponentially with the number of variables and clocks [3]. This problem is the main limitation of the technique, in the literature [14] [15] the model checking technique allows to verify timing properties on wireless networks composed of at most a dozen of nodes which is not sufficient for most of modern networks.

In the literature, most of the formal verifications of real-time systems rely on predictability of the behavior of the system. Nevertheless, in reality, unexpected errors such as packet losses can occur. In order to handle this issue, probabilistic formal methods have been proposed. Probabilistic model checking [16] allows to assess that a system model respect some probabilistic properties (for example, "with probability higher than 0.9995 the end-to-end delay is lower than 1 second"). This method have been applied to network protocol verification [15], but suffers from the same scalability problem as classic timed model checking. Network Calculus also possesses its probabilistic or stochastic counterpart [17] [18]. It allows to express stochastic delay bounds instead of deterministic ones. Nevertheless, it sufferers from the same drawbacks as the classic formalism (bounds might not be tight and it lacks a representation of the network dynamic).

To sum up, the presented methods to derive worst case delays in networks either do not scale up or do not provide tight bounds. In the past few years, the real-time computing community have made the same observation concerning the study of worst-case execution times of programs, and develop an alternative statistical approach [19] [5] based on EVT [6].

In the literature, EVT has been extensively used in very different contexts: extreme rainfalls [20], forest fires [21], wind speed [6], financial crashes [22] studies. EVT has also been used in the context of computer networks for the estimation of traffic peaks or bursts [23] [24] [25]. In [24], the author proposes to study the traffic on an Ethernet network in order to predict traffic peaks. The author shows that the gathered extreme deviations fit very well to a Generalized Pareto distribution as predicted by the theory. In another study [23], the authors apply EVT to the study of traffic throughput in wireless networks and show that the generalized EVT distribution is a better match for large deviation prediction than exponential, gamma or lognormal distributions. In [25] the authors fit Ethernet traffic throughput data to a Weibull distribution (also predicted by EVT). Nevertheless, in these applications of EVT to network traffic throughput, the authors arbitrary use 
only one of the two theorems of EVT and this choice is not clearly motivated. Moreover, the authors of these works do not focus on the study of large delays, but only on throughput.

Another interesting reference on the use of EVT in a networking context is [26]. The authors focus on opportunistic packet scheduling in wireless networks in which the scheduler chooses the node with the best current channel condition to transmit a packet in order to maximize the throughput. A delay analysis of this scheduling technique is proposed using EVT. The authors notably derive the packet delay distribution. Nevertheless, this distribution is not focused on large delays and it is then used to compute a metric based on the average delay. This metric is included in an alternative scheduling algorithm proposed by the authors which reduces the average packet delay, but no insight on large delays is provided.

As we stated previously, in order to find applications of EVT to the study of large delays in computer science, we have to look to the field of worst case execution times. Works such as [19] [5] make use of EVT to derive probabilistic worst case execution times. In this field, the Block Maxima (BM) is usually used and the data is fitted to the Gumbel distributions family.

The novelty of our work comes from the fact that we specifically discuss the application of EVT to delays in wireless networks. In particular, we discuss the validity of the hypothesis of EVT in this context. On the contrary of several papers of the literature, we explore the two available EVT techniques and discuss their pertinence to characterize large delays in networks. Up to our knowledge, this work represents the first attempt to apply EVT to the analysis of worst case delays in wireless networks.

\section{A brief introduction to EVT}

Extreme Value Theory have been developed during the 20th century and is now a well established tool to study extreme deviations from the average of a measured phenomenon [6]. EVT is built around two main theorems: the Fisher-Tippett-Gnedenko theorem and the Pickands-Balkema-de Haan theorem. As we detail below, the former is interested in the maximum value of a sequence of variables, whereas the latter focuses on the values of a sequence which are above a given threshold.

The Fisher-Tippett-Gnedenko theorem states that given $\left\{X_{1}, \ldots, X_{n}\right\}$ a sequence of independent and identically distributed (i.i.d.) variables, the 
distribution of $M_{n}=\max \left\{X_{1}, \ldots, X_{n}\right\}$ the variable representing the maximum value of the sequence converges (for large $n$ ) toward the extreme value distribution characterized by its CDF (Cumulative Distribution Function) denoted $G$ :

$$
G(x)=e^{(-1+\gamma x)^{\frac{1}{\gamma}}}, \text { with }-1+\gamma x>0
$$

with $\gamma$ a shape parameter of the distribution.

Depending on $\gamma, G$ belongs to one of these three distribution families:

- Frechet:

$$
G(x)=e^{-\left(\frac{x-m}{s}\right)^{-\alpha}}, \text { for } x>m
$$

if the distribution has a heavy tail;

- Gumbel:

$$
G(x)=e^{-e^{-\frac{x-m}{s}}}
$$

if the distribution has an exponential tail;

- Weibull

$$
G(x)=e^{-\left(-\left(\frac{x-m}{s}\right)\right)^{\alpha}}, \text { for } x<m
$$

if the distribution has a finite maximum;

with $m, s$ and $\alpha$ the distribution parameters and $\alpha>0$ in all cases.

The second theorem of EVT is the Pickands-Balkema-de Haan theorem. It states that given $\left\{X_{1}, \ldots, X_{n}\right\}$ a sequence of i.i.d. variables, the conditional distribution $F_{u}(y)=P\left(X_{i}-u<y \mid X_{i}>u\right)$ of each random variable $X_{i}$ of the sequence converges toward a generalized Pareto distribution for large $u$ :

$$
\left\{\begin{array}{l}
G(y)=1-\left(1+\frac{(y-m) \gamma}{s}\right)^{-\frac{1}{\gamma}}, \text { if } \gamma \neq 0 \\
G(y)=1-e^{-\frac{y-m}{s}}, \text { if } \gamma=0
\end{array}\right.
$$

with $\gamma$ the shape parameter of the generalized Pareto distribution.

Proofs for both of these theorems are provided in [6]. In this paper, we are interested in how to apply them to the study of large delays in wireless networks. To each theorem corresponds a method which can be applied to characterize the distribution of extreme variations of a phenomenon. For the first theorem the method is the Block Maxima (BM) method in which the sequence of measured data is divided into blocks and the maximum of each block is computed. The maxima data is then fitted to one of the three 
previously mentioned distributions. The second theorem of EVT corresponds to the Peak Over Threshold (POT) method. In this method, a threshold value is chosen (the choice of the value is a difficult question that will be discussed in section 5.4.3) and the data points which are above the threshold are collected and fitted to a generalized Pareto distribution. We can note that this description of EVT only considers univariate analysis, the interested reader might refer to [6] for a description of the multivariate case.

In the next section, we discuss how these methods can be applied to the study of delays in wireless networks.

\section{Applying EVT to the study of delays in networks}

In this section, we discuss the application of EVT for the study of worst case delays in wireless networks. By delays, we mean the measure of time between two events in the network. The delays studied in networks are of different kinds: end-to-end delays, one-hop delays, round-trip times, interpacket delays, etc. In this section we discuss the applicability of EVT to these measures. In section 5, in order to give an example of the application of EVT, we will focus on the case of inter-packet delays in VANETs.

\subsection{Gathering data}

The first element needed in order to apply EVT is a set of data which is a realization of the sequence of random variables $\left\{X_{1}, \ldots, X_{n}\right\}$ mentioned in the previous section. In our case it will be measures of time. Essentially, the data set is going to be similar as the one represented in Figure 1(a): a sequence of delays (y axis) measured at different points in time ( $\mathrm{x}$ axis). We can notice that unlike many data sets found in the literature [6] [20], delay measures are not regularly spaced in time because events in a network may not occur on a regular basis (notably because of the sporadic nature of data traffic).

We can also notice that, in this paper, we do not make special assumption on the data gathering technique. Nevertheless, in the case study section (section 5), we specifically use discrete-event simulation.

As stated in section 3, in order to apply the EVT theorems the data variables have to be i.i.d.. We have to remark that this hypothesis may influence the data gathering strategy. This issue is discussed in the next subsection and illustrated in section 5 . 


\subsection{The i.i.d. hypothesis}

According to Fisher-Tippett-Gnedenko and the Pickands-Balkema-de Haan theorems, to apply EVT, the sequence of variable must be independent and identically distributed. The question then is: can it be the case for delays in wireless networks?

Let's first consider two packet flows $\mathrm{A}$ and B, following different paths. Can we assume that their packet end-to-end delays, measured at the destinations, are identically distributed? We would tend to answer yes if the network environment and measure conditions are the same for both A and B (same channel conditions, same protocols, fair access to the medium, same class of traffic, same number of hops, etc). This leads us to believe that to apply EVT on network delays we have to be careful not to have great disparities in the studied network like very dense and very sparse areas in the same network.

Concerning the independence hypothesis, we can argue that the hypothesis will hold if we consider networks in a globally stable state. For example if congestion in the network changes too much over time we will observe successive delay measures as correlated in subsets of the sequence. On the contrary if the parameters which influence the network are stable, even if we have local correlations, the sequence of measured delays in the whole network should appear as uncorrelated.

These qualitative statements will be experimentally verified in the case study in Section 5. In the next subsections, we discuss the applications of the two EVT techniques: BM and POT to delays in networks.

\subsection{Block Maxima}

As explain in section 3, the Block Maxima method corresponds to the Fisher-Tippett-Gnedenko theorem. It focuses on the distribution of the maxima of sequences of measures. In this section we discuss the practical details of the application of BM to the study of the maximum delay in wireless networks.

The principle of the BM technique is to divide the data sequence into blocks and to take the maximum of each block. In the literature, a block is often defined as a time interval [6]. Figure 1(b) shows an example of application of BM: it is the same data set as Figure 1(a) divided into blocks of size 20 seconds. In each block the maximum is boxed. Then, according to the Fisher-Tippett-Gnedenko theorem the sequence of block maxima must converge to an extreme value distribution. The block maxima sequence can 


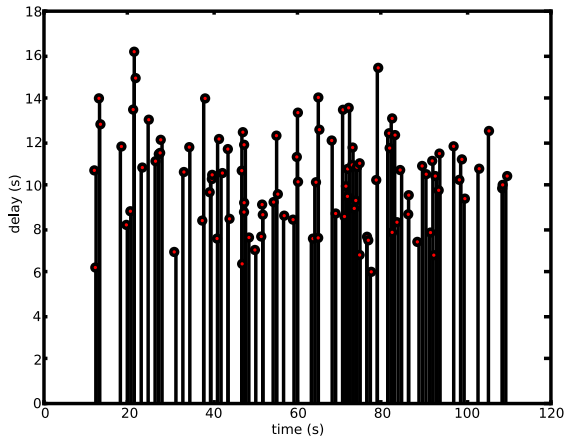

(a) Raw data

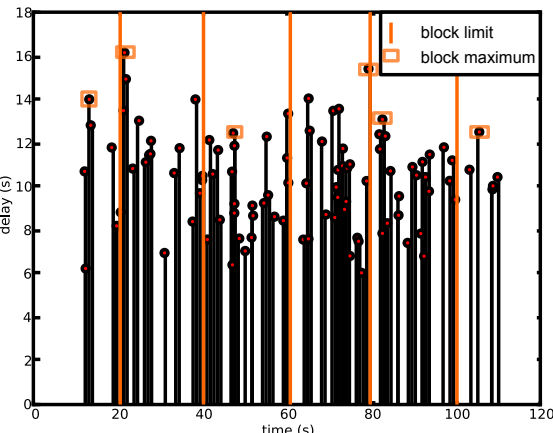

(b) Block Maxima

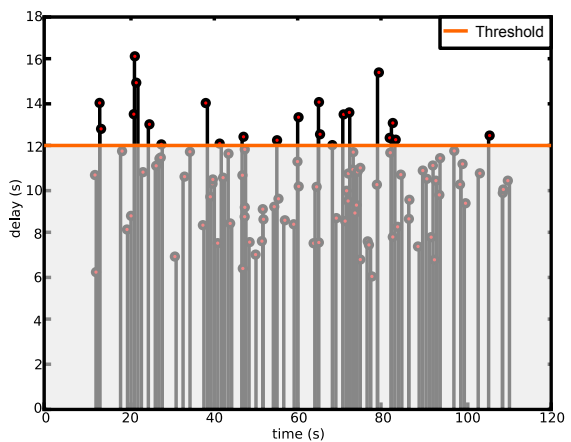

(c) Probability Over Threshold

Figure 1: Illustrative example data set

thus be fitted to one of the Gumbel, Weibull or Frechet distributions. The type of distribution the data will converge to is difficult to predict [20]. In the case study section, we thus try the three distributions and conclude thanks to the statistical tests described in Section 4.5.

We can note that the width of the block is an important parameter: it must be large enough to contain sufficient data in order to apply the FisherTippett-Gnedenko theorem (which concerns asymptotic behavior) and not so large to provide a sufficient number of maximum values to be fitted to a distribution. Nevertheless, "sufficient amount of data" is not clearly defined in the literature [5]. In practice, in the case of discrete event simulation, a scenario can easily be replayed a large number of times with different random generator seeds in order to produce an important amount of data. This question will be further discussed in section 5.3 while applying the BM method to the VANET safety application case study. In particular, we 
investigate how the size of the block influences the parameters of the fitted distribution and the goodness of fit.

\subsection{Peak Over Threshold}

The Peak Over Threshold method is an application of the PickandsBalkema-de Haan theorem. It focuses on the distribution of a variable knowing that this variable takes values greater than a threshold. In order to apply this method, we have to define a threshold and keep only the values of the data sequence which are above the threshold as depicted in Figure 1(c).

First, we have to notice that usually the POT method makes a much more efficient use of the data set [5]. Indeed, in the example of Figure 1(a) where we had only 6 values to fit with a distribution in the case of BM (see Figure 1(b)) we now have 22 values for the fitting with the Generalized Pareto distribution, as can be seen in Figure 1(c). Nevertheless, the choice of the threshold is not trivial and has not received much attention in the literature [6]. Similarly to the size of the block for the BM method, the value of the threshold must be chosen sufficiently high so that the Pickands-Balkema-de Haan theorem can be applied, but not so high so there is sufficient data points to be fitted to the Generalized Pareto distribution. This issue is investigated in Section 5.4 where the influence of the threshold on the parameters of the fitted distribution and the goodness of fit is investigated.

Moreover, in the POT case, the interpretation of the result is not as straightforward as the BM case. In the latter case, we directly obtain the probability distribution of the maximum delay value so it directly provides us with the probabilistic worst case delay. In the POT case, we obtain the probability distribution of the delay knowing that the delay already exceeds a given threshold.

These issues will be investigated and discussed further in section 5.4.

\subsection{Fitting technique and statistical test}

In the two previous subsections, we have described how to retrieve extreme value data from the original data set. We then have to fit this extreme value data to one of the extreme value distributions predicted by the extreme value theorems and assess that the fitted distribution is an acceptable representation of the data thanks to a goodness-of-fit statistical test. In this paper we use the Maximum Likelihood Estimation (MLE) technique to estimate the parameters of the distributions, and the Pearson's chi-squared test and quantile-quantile plots to assess the goodness-of-fit [2]. 
The MLE method consists in finding the vector of parameters for the fitted distribution (in our case Frechet, Weibull, Gumbel or generalized Pareto) such that the likelihood function is maximized. In this paper, in order to solve this optimization problem we use the Nelder-Mead method [27] (more precisely, the implementation from the scipy.optimize Python package [28]).

Once the optimal distribution parameters are obtained, we have to verify that the fitted distribution is actually a convincing representation for the data. For that purpose, we use two tools: the Pearson chi-squared test and quantile-quantile plots [2]. The former is a statistical test which assesses if there is a statistical difference between an observed data frequency distribution and a theoretical distribution. In this paper, we use the implementation from [29]. We can note that this test's statistic offers a quality indicator for the fit which can be used in the case several distributions pass the chi-squared test as in [20].

The quantile-quantile plot [2] (or Q-Q plot) is a tool which allows to graphically compare two distributions. In our case, it consists in plotting the quantiles of the collected data against the quantiles of the fitted distribution. If the two distributions match, we should obtain the relation $x=y$. As described in [6] and [2], the plot consists in a set of points $(x, y)$ where $x \in\left\{x_{1}, \ldots, x_{n}\right\}$ with $\left\{x_{1}, \ldots, x_{n}\right\}$ the ordered set of data points (in increasing order) and $y=F^{-1}\left(\frac{i}{n+1}\right)$ with $n$ the number of data points, $i=1, \ldots, n$ and $F^{-1}$ the inverse CDF of the fitted distribution. If the Q-Q plot points do not fall on the $x=y$ line, several interpretations are possible [2]. In our case, if the points deviate above the $x=y$ for a $x$ given range (the slope of the curve formed by the point increases), it can be interpreted as an excess of data points compared to the theoretical distribution in this range. On the contrary, if the slope decreases, it indicates a lack of data points compared to theoretical predictions.

\section{Case study : inter-beacon delays of VANET safety applications}

In this section, we apply the presented method to a concrete case: the study of the inter-beacon delays of VANET safety applications. We first introduce the context of safety applications for VANET, we then present the simulation setup and apply BM and POT methods to the simulation results. For each method, we discuss the cases in which it could or could not be applied. 


\subsection{Safety applications for VANET}

The safety applications are seen as an essential motivation for the deployment of VANETs [1]. The main goal of these applications is to avoid car crashes. We can cite few examples of safety applications [30]:

- traffic signal violation which alerts neighbor cars when a user does not stop at a red traffic light;

- electronic brake which alerts neighbor vehicles when a car performs an emergency braking;

- on-coming traffic warning which notifies the driver of on-coming traffic during overtaking maneuvers.

For more examples, the interested reader may refer to a report of the U.S. National Highway Traffic Safety Administration [30], which provides a wide overview and requirements of safety applications, or to Chapter 2 of [1] for a more concise description.

The main building block for safety applications is the exchange of periodical one-hop broadcast messages among neighbor vehicles. These messages are called beacons or heartbeat messages in the literature and contain at least information on the cars position, direction and speed [31] [8]. In the architecture defined by the ETSI ITS standard [32], these messages are called Cooperative Awareness Messages (CAM). In the case of the IEEE WAVE standard [33], they are named Basic Safety Messages (BSM). When receiving these messages, each car can build a representation of its environment, analyze the current situation and predict dangerous situations. The period, latency and scope (or range) of the beacons depend on the requirements of the application. For example, according to [1] the electronic brake application needs a beacon frequency of $10 \mathrm{~Hz}$, a maximum latency of $100 \mathrm{~ms}$ and a range of $200 \mathrm{~m}$ to be effective.

In VANETs, these beacon messages are exchanged among cars using Direct Short Range Communications [1]. Both U.S. and European standards use variants of IEEE 802.11p [9] which is an amendment of the widely used IEEE 802.11 standard. Since 802.11 is regarded as the standard of choice for the future deployment of vehicular network, we focus on beaconing using this standard. The standards (IEEE WAVE and ETSI ITS) define several communication channels and a unique control channel $\mathrm{CCH}$. The $\mathrm{CCH}$ is envisioned to support the beaconing traffic [1]. 
In this context, the vehicles broadcast their safety beacons on a common channel. The main differences between 802.11 and $802.11 \mathrm{p}$ are pointed out in [9]. The broadcast procedure remains unchanged and is described as follows. The time is divided into slots. First the sender senses the medium. If it is free, it transmits the beacon. If it is occupied: it picks a random number $N$ uniformly in $[0, C W]$ (with $C W$ the initial contention window value), then senses the medium and decrements $N$ for each free slot. When $N=0$, it transmits the beacon. We can note that unlike the unicast case, there are no retransmissions and thus the $C W$ value is not updated [31].

With this procedure, messages can collide and thus the neighbors of a sender may not receive some of the beacons. Moreover, other messages can be lost because of the unreliability of the radio links. These message collisions and losses increase the time period between consecutive beacons at the receiver side. In this case, vehicles may not be able to construct an up-todate representation of their environment and prevent efficiently car crashes. Since, the quality of the environment's representation depends on the freshness of the information about the neighbors, the inter-beacon delay is thus a crucial parameter for critical safety applications. In this paper, we therefore choose to study the inter-beacon delays in VANETs in a realistic simulation environment which is described in the next subsection.

\subsection{Production of the data: the simulation setup}

There exist many studies [1] which focus on beacon messages for critical applications. The aim of the one we develop in this paper is twofold: it allows to show the applicability and usefulness of the EVT approach for the study of delays in wireless networks, and it allows to reproduce results of the literature [1] [31] [34] [8] [35] (notably the fact that 802.11 is not adapted for reliable beaconing) about beaconing in VANETs with 802.11 with a realistic mobility scenario [7].

We use the discrete-event simulator ns2 [36] to perform the simulations, the main simulation parameters are described in Table 1 . The simulation setup is actually quite classic, we thus focus on the description of the realistic mobility trace and how we use it in the simulator.

The mobility trace we use in the simulations is a realistic micro-mobility trace of the city of Cologne generated by Uppoor and Fiore [7]. The trace covers a $400 \mathrm{~km}^{2}$ area and a period of 24 hours and contains about 700000 vehicle travels. In this paper, we use the part of the trace available online which covers the 6am-8am period. In Figure 2, we plot the instantaneous 


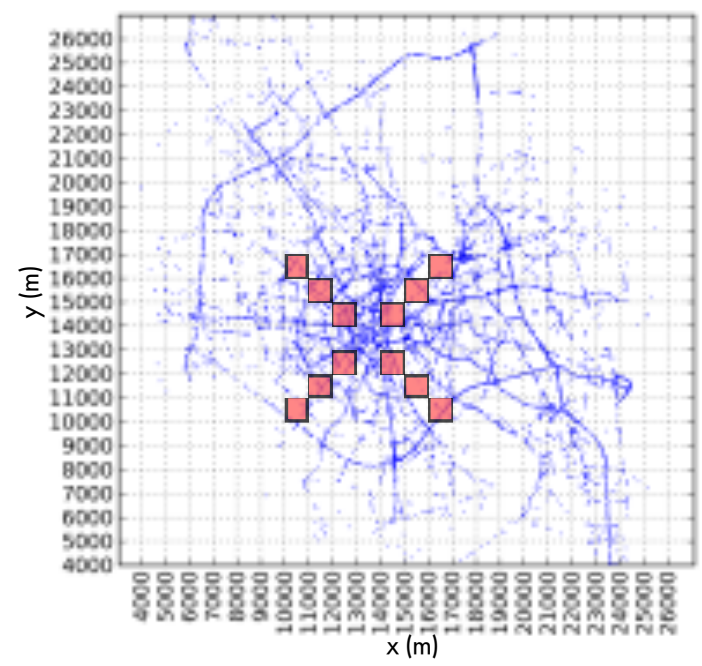

Figure 2: Cologne data set

positions of the nodes at 8am. Each point corresponds to a vehicle position in the coordinate system provided by the trace (the coordinates are expressed in meters). The trace from 6am to 8am contains more than 300000 different vehicles, it is thus not possible to simulate the whole scenario in ns2. As we are interested in local communications (one-hop broadcasts), we decide to divide the network and restrict the simulations to $1000 \times 1000 \mathrm{~m}$ squares. The considered squares are highlighted in Figure 2. They contain different types of road traffic (fluid traffic, traffic jams, etc) and different vehicle densities. Even with this space division of the trace, the number of cars in one square can reach several hundreds and, in some cases, the simulations are either very long (dozens of hours) or not possible (the memory of the machine used for the simulation is not sufficient). We thus divide again the trace, but this time into time intervals. Instead of having 2 hours of simulated time we produce subset traces of 200 seconds. Each of the time blocks also contains different traffic conditions, since the traffic changes over time in the trace.

In each simulation, we monitor the inter-beacon delay as well as the emitter-receiver distance. The beacons are emitted at $10 \mathrm{~Hz}$ corresponding to a $0.1 \mathrm{~s}$ beacon emission period. The inter-beacon delays we are interested in are measured at the receiver, because it corresponds to the time a node spends without awareness of its neighbors. Each node computes these delays for every of its neighbors. A simulation typically provides around 2.5 millions 
measurements of inter-beacon delays (it varies depending on the number of cars present during the simulation).

\begin{tabular}{|l|l|}
\hline Parameter & Value \\
\hline Bitrate & $6 \mathrm{Mbps}$ \\
Transmission power & $10 \mathrm{dBm}$ \\
Preamble Capture & $\mathrm{ON}$ \\
Simulation area & $1000 x 1000 \mathrm{~m}$ \\
Beacon size & 400 bytes \\
MAC and Phy & $802.11 \mathrm{p}$ \\
Propagation model & Nakagami $\mathrm{m}=1$ \\
Beacon frequency & $10 \mathrm{~Hz}$ \\
\hline
\end{tabular}

Table 1: Simulation parameters.

In the following sections we detail how the simulations are run and the data processed in order to extract the inputs needed by the BM and POT techniques. We apply both techniques and discuss their pros and cons.

\subsection{Block Maxima}

In this subsection, we apply the BM method to study the maximum interbeacon delay distribution in VANETs.

\subsubsection{Data set}

For the BM technique, we need to divide the data into blocks and retrieve the maximum of each block. We choose to use three different ways of producing the data which is then fitted to EVT distributions:

1. The first one consists in taking the maximum of each block of 200 seconds (corresponding to one run of the simulator) for the whole trace duration and all the highlighted squares in Figure 2.

2. In the second one we take only one square and one portion of time and re-run the simulation for that particular square and time block several times.

3. The third case is the same as the second but we change the order of the beacon start of the nodes at each run (in the second case, the order of beacon start dates is generated randomly once and the same order is repeated in every simulation). 

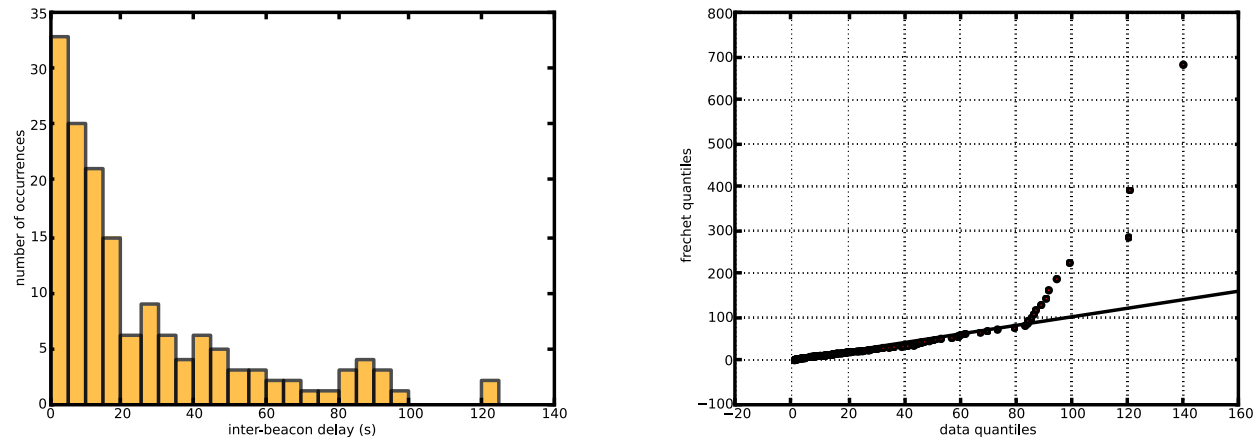

(a) Histogram of the maximum inter-beacon (b) Q-Q plot: data against Frechet distribudelays tion
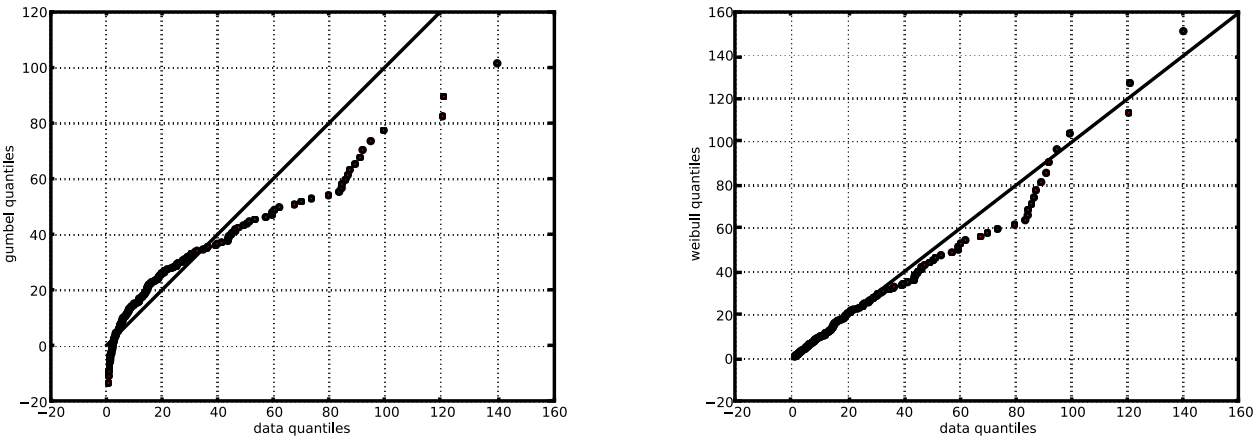

(c) Q-Q plot: data against Gumbel distribu- (d) Q-Q plot: data against Weibull distribution tion

Figure 3: Results for all the squares and blocks and 500m range

These different setups allow us to understand how disparities in the simulation data affects the applicability of the BM method. Indeed, in the first case, the data collected comes from various situations in terms of car traffic amount, network density, etc. Whereas in the two last cases the blocks are more similar to one another (in the second data set there are more correlations between the runs than in the third data set because the beacon start dates are the same).

For setup 1, we run simulations for 13 successive blocks of 200 seconds for each of the 12 considered squares. We take the maximum inter-beacon delay (over around 2.5 million values) for each run and thus obtain 156 values. For setup 2 and 3 we run 300 simulation of the considered block, so we obtain 300 
maximum values. In the results presented in the following sections, we only consider the receivers in a 500 meters range from the sender. Even though longer communication ranges are observed given the simulation parameters (see Table 1), we choose to focus on the delay of beacons received from neighbors less than 500 meters away which are the more relevant for safety applications [1]. We thus filter out inter-beacon delays for ranges greater than 500 meters from the simulation results. We also investigate the maximum inter-beacon delay distributions for shorter than 500 meters ranges.

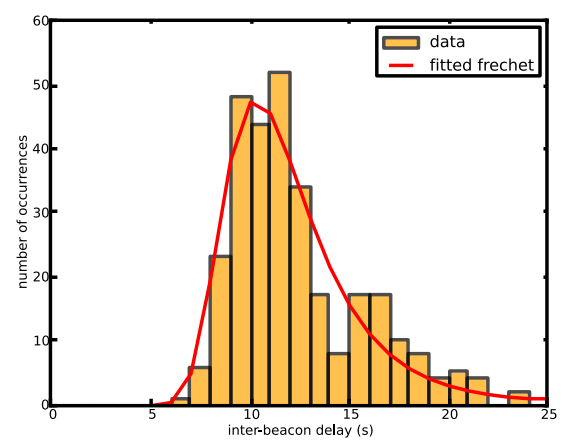

(a) Histogram of the maximum inter-beacon delays

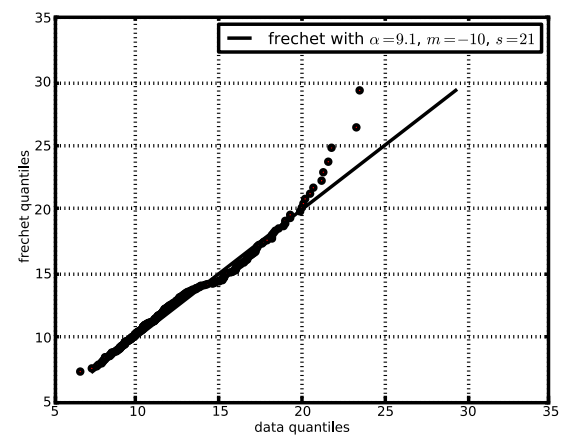

(b) Q-Q plot: data against Frechet distribution

Figure 4: Results for one square during 200 seconds

\subsubsection{Maximum inter-beacon delay distribution}

Figure 3(a) is a histogram representation of the maximum delays for the first data set. First, we have to note that the measured maximum interbeacon delays are very large compared to the inter-beacon emission period (0.1 seconds), but we have to keep in mind that these are maximum delays and each value accounts only for the maximum of 2.5 million delay measurements as explained in the previous section. The study of the simulation traces shows that these large values are due to two main reasons: the mobility and the broadcast scheme used. When a node broadcasts its beacon using 802.11p, it cannot detect collisions. Indeed, collisions happen at the level of the receivers and the sender does not know which of its neighbors will actu-

ally receive the packet and does not wait for acknowledgments in the case of broadcasting. Moreover, broadcast messages are subject to the hidden terminal problem since the RTS/CTS messages are not used for broadcast. These 
problems have been highlighted several times in the literature [1] [31]. In order to better comprehend the observed long maximum delays, let's consider the following scenario: first, a node 1 periodically receives a beacon from one of its neighbors 2 , then another node, node 3 moves in the neighborhood of 1 and the beacons from 3 collides with those of 2 (in the case of the hidden terminal problem the beacons may constantly collide), then node 3 moves out of range of node 1 again. In this scenario, when node 3 at last get out of the range of node 1 , it puts an end to the collisions with beacons from node 2. When node 1 receives the first beacons from node 2 after node 3 went out, the inter-beacon delay is approximately equal to the duration of the presence of node 3 in the range of node 1 . In the Cologne trace case, this delay can be of the order of tens of seconds as we observe in the simulations results. Even if these scenarios are very rare, they have to be taken into account in the scope of critical applications such as road safety applications.

Let's now focus on the fitting of the data of the first data set to the different extreme value distribution families. Figures 3(b), 3(c) and 3(d) respectively represent the Q-Q plots of the fitted Frechet, Gumbel and Weibull distributions (fitting has been realized using the MLE method described in section 4). First we observe that none of the distribution fits well to the data. Indeed, none of the graphs show a $x=y$ curve. Nevertheless, we remark that in the Frechet case the curve is piecewise linear which seems to indicate that there are linear relations between the data quantiles and the fitted Frechet distribution quantiles. In the case of the fitted Weibull distribution, we observe that for $x$ and $y$ lesser than 60, the points are approximately on the $x=y$ curve.

The fact that the Q-Q plots are piecewise, seems to indicate that actual maximum inter-delay distribution is multimodal. In fact, from the simulation data, we observe that the different modes correspond to different areas (squares) of the network and different time periods. We conclude that the EVT hypothesis which states that the set of inter-beacon delays are identically distributed does not hold for this data set and thus it is not possible to apply EVT. As a matter of fact, the results of the chi squared tests for all three EVT distributions for this data set are negative as shown in Table 2 (the tests are performed with a 0.05 significance level). We can add that these observations holds for shorter ranges as well. For instance, we also observe multimodal histograms when considering only beacons with a range shorter than 300 meters.

Figures 4(a) and 4(b) respectively depicts the frequency plot of the inter- 


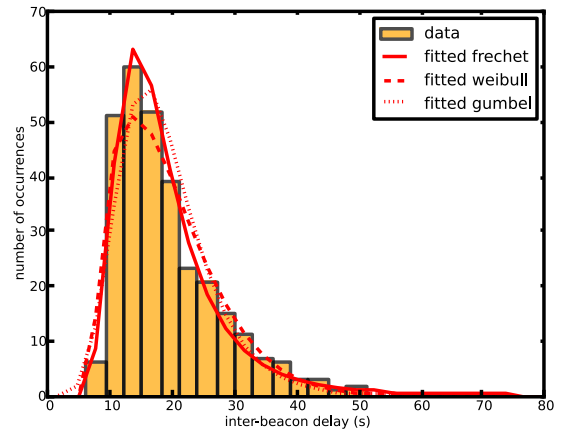

(a) Histogram of the maximum inter-beacon delays

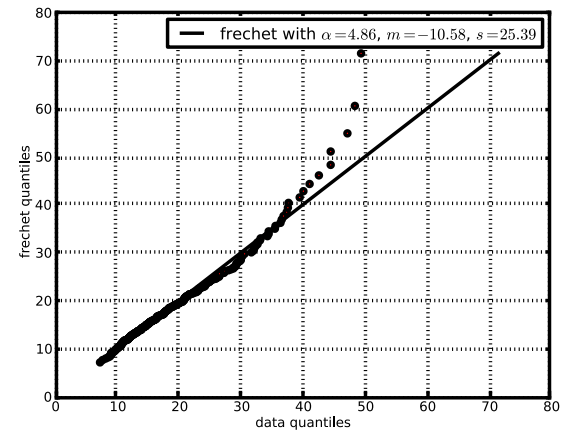

(b) Q-Q plot: data against Frechet distribution

Figure 5: Results for one square during 200 seconds with random beacon start at each run

\begin{tabular}{|c|c|c|c|}
\hline & Data set 1 & Data set 2 & Data set 3 \\
\hline Frechet & $\times$ & $\checkmark$ & $\checkmark$ \\
\hline Gumbel & $\times$ & $\times$ & $\checkmark$ \\
\hline Weibull & $\times$ & $\times$ & $\checkmark$ \\
\hline
\end{tabular}

Table 2: Chi-Squared test

beacon delays with the expected frequencies from the fitted Frechet distribution and the Q-Q plot of the data against the fitted distribution for the second data set. As stated in section 5.3.1, this set consists of one square and one portion of 200 seconds run 300 times. The chosen square is defined as $x \in[11000,12000]$ and $y \in[11000,12000]$ (cf. Figure 2) and the considered time block is from 1200 to 1400 seconds of the original trace. In this case, as summed up in Table 2, only the fitted Frechet distribution successfully passes the Pearson chi squared test. The Q-Q plot (Figure 4(b)) shows that the fit is good for the lowest values and of a lower quality for the highest values. We can also still discern at least two modes in the frequency distribution in Figure 4(a) (also visible in Figure 4(b), because the points lie over the $x=y$ curve for $x<15$ and then under until approximately $x=18$ ).

Figures 5(a) and 5(b) present the results for the third simulation setup (the third data set): it is the same as the previous (one square for one 200 second interval run multiple times) but the nodes are starting their beacon emission at different dates in each simulation. The starting dates are in fact uniformly distributed in the first second of the simulation. The results 
presented in Table 2 indicate that for this data set, all three fitted EVT distributions pass the chi squared test. Figure 5(a) depicts the frequency plot of the inter-beacon delays with the expected frequencies from the three EVT distribution. In Figure 5(b), we present only the Q-Q plot for the fitted Frechet distribution because even if all the distributions pass the chi squared test, the Frechet distribution is the best fit (the one with the lowest test statistic). In this Q-Q plot, we observe, as in the last, that the fit is good for the lower values and less good for higher values. Nevertheless, for this data set, we do not observe multimodal tendency which seems to indicate that the identically distributed hypothesis holds.

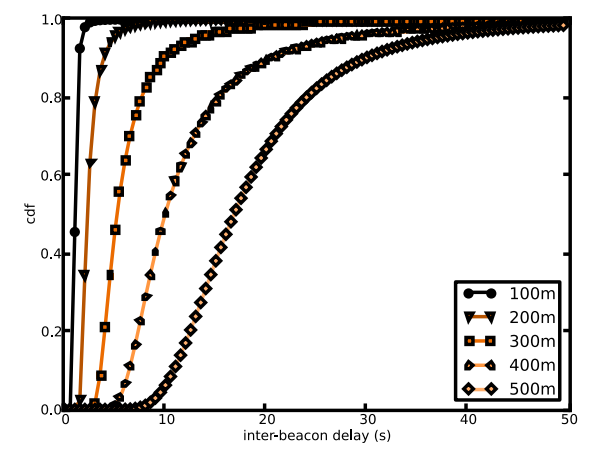

Figure 6: CDF for different beacon ranges

All the results we have presented so far are for a 500 meters range: we compute the inter-beacon delays for receivers within 500 meters of the sender. Nevertheless, for most of critical safety applications, the range of beacon emission can be much lower [30]. In Figure 6, we plot the CDFs of fitted Frechet distribution for different ranges. The CDF of the maximum delay express the probability that the maximum delay is under a given value. It corresponds to the probabilistic worst case delay. Thanks to this probabilistic worst case delay, the system designer can state that, for example, "the probability that the maximum inter-bacon delay in a 100 meters range around the sender is less than 5 seconds is $0.9995 "$. Whether this probabilistic delay bound is sufficient or not depends on the considered application. In Figure 6, we can notice that the CDF for higher ranges is lower. This means that the probability that the maximum delay is over a given value is higher for higher ranges. This can be explained by two phenomena: the higher probability of packet loss at longer distances and the hidden terminal problem as explained 
above in this section.

\subsubsection{Impact of the block width}

As stated in section 4.3, the width of the block from which the maximum value is extracted can have an impact on the quality of the probabilistic bound obtained when applying EVT. If it is too narrow, the Fisher-TippettGnedenko theorem might not be applicable. If it is too wide, not enough data is available for the fitting with the EVT distributions. Indeed, the MLE technique requires a sufficient amount of data in order to obtain robust models. In [37], the author recommend to use at least 100 values for MLE. In our case, the number of values depends on the block width: for the same simulation duration, a larger block width involves a smaller set of maximum values.

Figures 7(a) and 7(b) depict the Q-Q plot of the data against fitted Frechet distributions respectively for 100 seconds blocks and 400 seconds blocks. In order to produce the data sets corresponding to these block sizes, we used data set 3. We divided the 200 seconds block in two for the 100 seconds block and united two 200 seconds blocks for the 400 seconds blocks. For the 200 seconds blocks, the number of data points (measured delays) is about 2.5 millions. It is thus half that number for 100 blocks and twice that number for 400 blocks. The number of extracted maxima is greater when the block is smaller : in our case, it is 300 values for the 200 blocks, 600 for the 100 blocks and 150 for the 400 blocks. Following the recommendations of [37], we did not experiment with larger block width. Figures 7(a) and 7(b) can be compared with the 200 seconds block case for the third data set in Figure 5(b). First we have to notice that the 100 seconds and 200 seconds cases (respectively Figures $7(\mathrm{a})$ and 5(b)) are very similar: the fit is good for the first quantiles and less for higher quantiles (we can note that in every case, the fitted distribution passes the chi squared test). Interestingly, in Figure 7(b), we observe that the fit is better for 400 seconds blocks. It can be explained by the fact that the Fisher-Tippett-Gnedenko theorem applies for large blocks of data (blocks with high number of values). Because the theorem establishes a convergence to an extreme value distribution when $n$ goes to infinity, $n$ being the size of the sequence of random variables from which the maximum is taken.

Figure 7(c) shows the CDF of the fitted Frechet distributions for different block sizes. We observe that a greater block size produces a higher probabilistic worst case delay: by higher we mean that for a given probability 

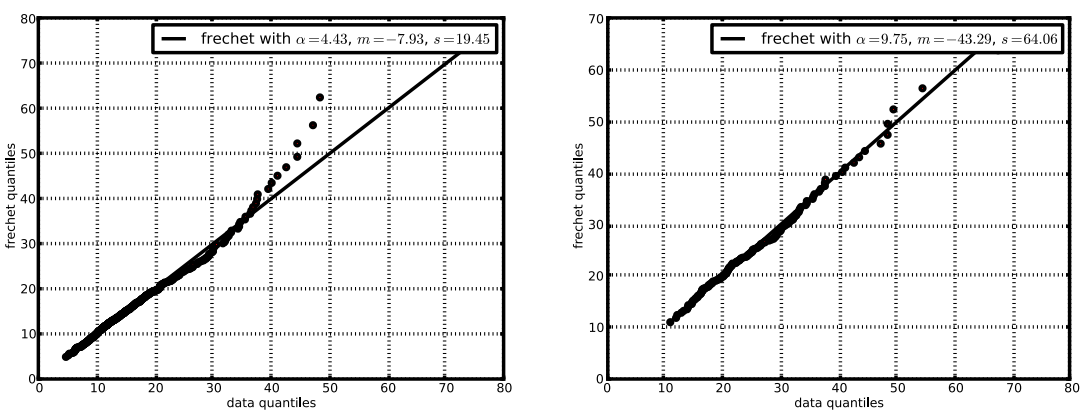

(a) Q-Q plot for 100s block size (data against Frechet distribution)

(b) Q-Q plot for 400s block size (data against Frechet distribution)

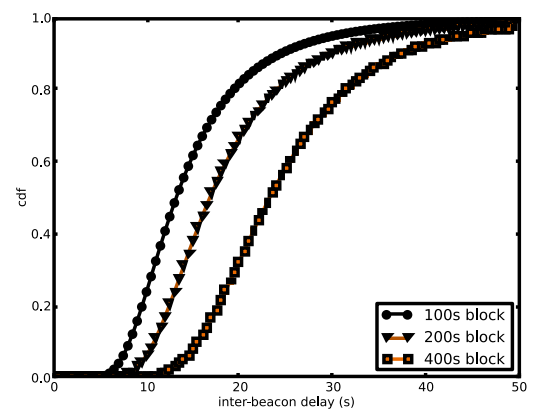

(c) Comparison of the maximum delay Frechet distributions for different block sizes

Figure 7: Results for different block sizes

threshold, the delay is greater. For example, if we consider a 0.8 probability to obtain a worst case delay under a given value, for the 100 seconds block case, this value is around 19s and for the 400 seconds block case, it is around 30s. This means that if the chosen block is too narrow, the obtained probabilistic worst case delay might be optimistic.

In the following sections, we move to the application of the POT method to our VANETs simulation results. We then discuss the possible interpretations and uses for the EVT results for both methods.

\subsection{Peak Over threshold}

In this section, we apply the POT method to the study of high interbeacon delays in VANETs. This method is the application of the Pickands- 


\section{Balkema-de Haan theorem.}

\subsubsection{Data set}

To avoid the applicability problems encountered when applying BM to the three considered data sets, in the POT case, we use only the third data set depicted in section 5.3.1 (one square, one time block, simulation ran 300 times). From this data set, we keep only the values which are over a chosen threshold to fit it to a generalized Pareto distribution.

We first choose the threshold to be 10 seconds. It seems a reasonable choice since we actually observe values above 10 seconds and that 10 seconds is very high compared to the theoretical inter-beacon delay which is 0.1 second. Then, in section 5.4.3, we change the value of the threshold in order to observe its effect on the quality of the fit with a generalized Pareto distribution.

\subsubsection{Inter-beacon delay distribution for delays over a threshold}

First we have to notice that even if the data fitting process and statistical test is the same as the BM method, the result given by the fitted distribution does not have the same interpretation. Indeed, the distribution obtained from the BM method is the distribution of the maximum delay, whereas the distribution obtained from the POT method is the distribution of the delay knowing that this delay is already over a given threshold. This distinction has already been noticed in section 4.4 and will also be commented in section 5.6 .
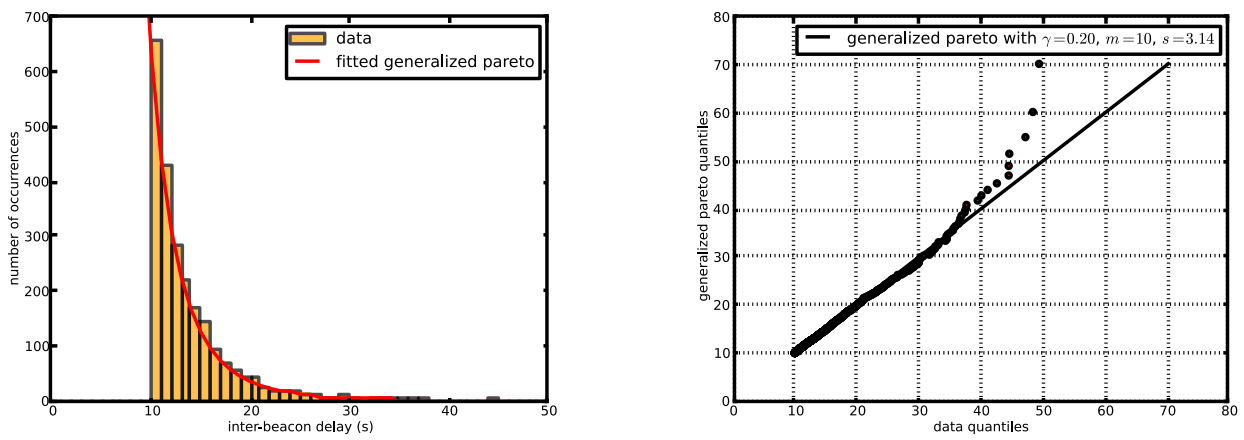

(a) Histogram of the inter-beacon delays (b) Q-Q plot: data against generalized over $10 \mathrm{~s}$ Pareto distribution

Figure 8: Results for The POT method with a threshold of 10 seconds 
Figures 8(a) and 8(b) depict respectively, the frequency plot of the data with the expected frequencies computed from fitted generalized Pareto distribution and the Q-Q plot of the data quantiles against the fitted distribution data quantiles. In Figure 8(b), we observe that the quantiles of the fitted generalized Pareto distribution are a very good match for the ones of the data for values under $x=35$, then the fit is of a lesser quality. Nevertheless, the fitted distribution passes the Pearson chi squared test. The fitted general Pareto distribution can thus be considered as a good model for the tail of the inter-beacon delays for this data set.

\subsubsection{Impact of the threshold value}

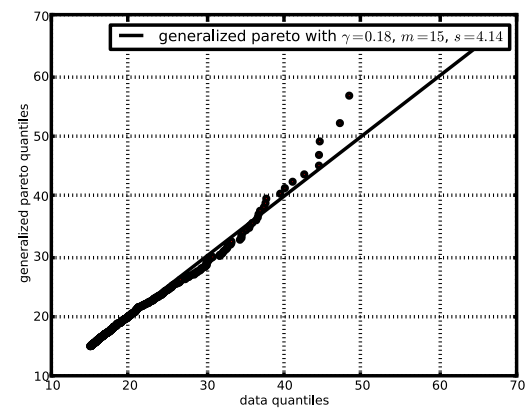

(a) Q-Q plot for $15 \mathrm{~s}$ threshold (data against generalized Pareto distribution)

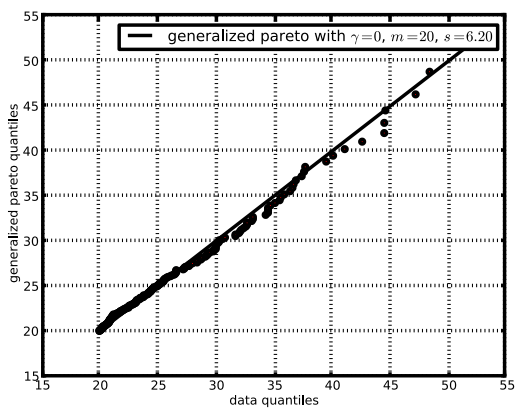

(b) Q-Q plot for 20s threshold (data against generalized Pareto distribution)

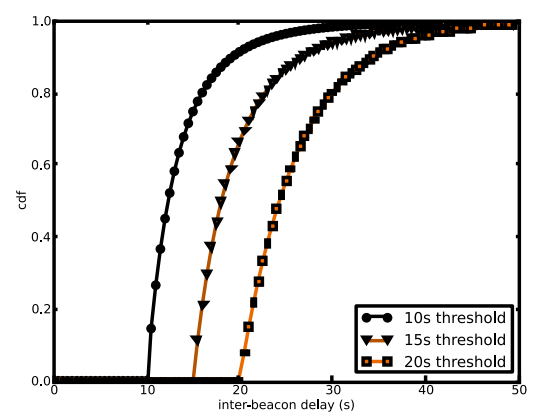

(c) Comparison of the delay generalized Pareto distributions for different thresholds

Figure 9: Results for different thresholds 
In this section, we investigate the impact of the threshold value on the quality of the fit. In this case, the threshold must be high enough for the Pickands-Balkema-de Haan theorem to be applicable but not too high in order to have a sufficient amount of data to feed to MLE (at least 100 values, as mentioned in Section 5.3.3). Figures 9(a) and 9(b) represent respectively the Q-Q plots of data against the fitted generalized Pareto distribution for $15 \mathrm{~s}$ and $20 \mathrm{~s}$ thresholds. First, we have to notice that the higher the threshold is, the lower the number of data points is. In our case, the number of delays over 10, 15 and 20 seconds is 2380, 619 and 208 respectively. We do not use higher thresholds in order to keep the number of values sufficiently high. Figures 9(a) and 9(b) can be compared to Figure 8(b) which is the Q-Q plot for a 10s threshold. We observe that the match between the data and the theoretical generalized Pareto distribution is better when the threshold increases, especially for high quantiles values (points are closer to the $x=y$ line).

Figure 9(c) is the plot of the CDFs of fitted generalized Pareto distributions for different thresholds. We cannot directly compare the distributions for values under 20 seconds, because it is the value of the highest threshold. Nevertheless, we observe that the models for higher threshold are more conservative because the curves do not cross each others and the CDF for higher thresholds converge more slowly to one. Since the distributions for higher thresholds are a better match for the data, we conclude that taking a too low threshold value can lead to underestimate the probability to observe a given high delay.

\subsection{Comparison of the model with new measurements}

Until this point, we have only been interested in the capacity of the EVT model to represent some gathered data. The natural question then is: how good the model is to predict new data? In [24], the author fits generalized Pareto Distribution to a subset of the available data and shows that the fitted distribution is a good model for the rest of the data set. In this section, we perform the same experiment but for the BM technique and instead of dividing the data set, we produce more data to compare to the model.

As we observed in Section 4.3, we can only produce a good quality EVT model for homogeneous parts of the network. Therefore, in this section, we compare the theoretical model obtained with the 400 seconds block in Section 5.3.3 with the data from simulation of the same square as data set 3 , but at different times. In order to produce this data, we ran simulation on this 
square at different time intervals of the trace with the same setup as the previous simulations (cf. Section 5.2). As in Section 5.3.3, we unite two 200 seconds trace intervals in order to get the 400 seconds block.

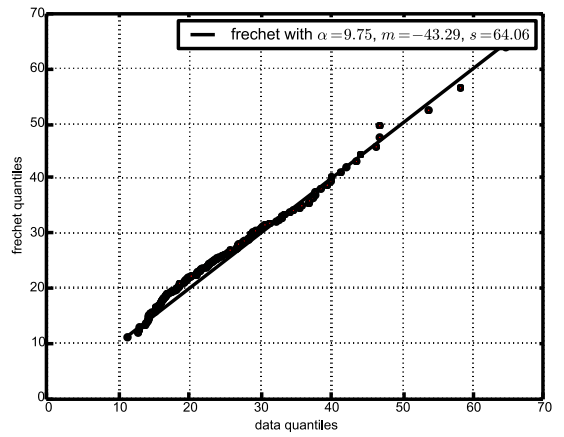

(a) Q-Q plot: data against Frechet distribution

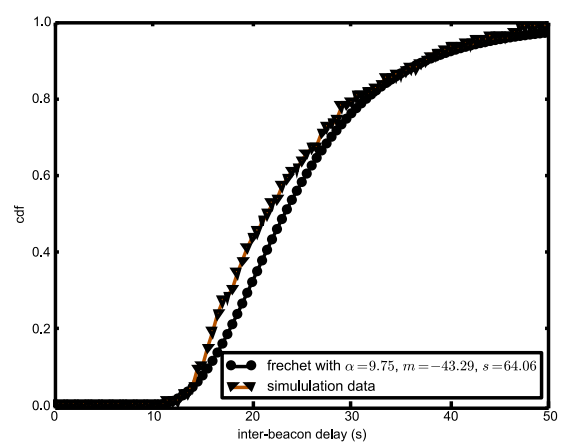

(b) Comparison of the theoretical and empirical distributions

Figure 10: Comparison with the following time interval

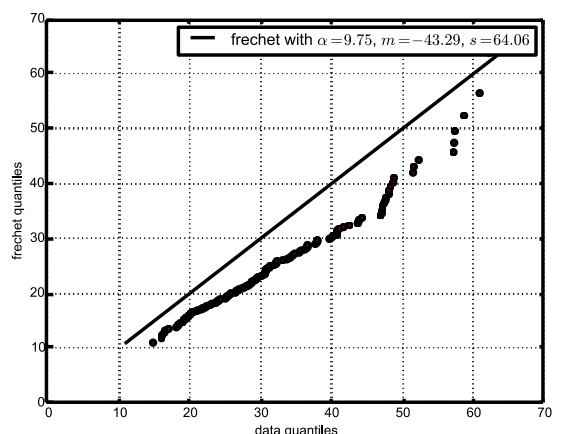

(a) Q-Q plot: data against Frechet distribution

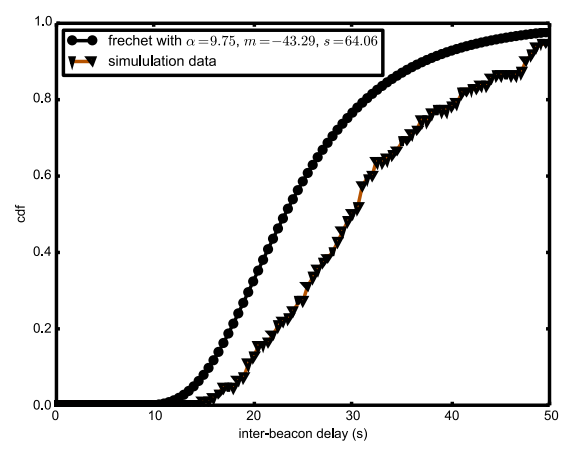

(b) Comparison of the theoretical and empirical distributions

Figure 11: Comparison with a later time interval

Figs. 10(a) and 10(b) depict a comparison of the data with the model for the time interval following the one used to produce data set $3: t \in$ $[1400,1600]$. In this interval, the traffic and mobility conditions remains very similar the ones used obtain the model in Section 4.3 so this model remains relevant. Indeed, in Fig. 10(a) which represent the Q-Q plot of the Frechet 
model versus the data collected, we observe a good match. Moreover, the Pearson chi squared test is positive. Fig. 10(b) shows the theoretical and empirical CDFs. We observe that they are very close and that, in this case, the model is conservative (the curve of the model is under the empirical curve).

Figs. 11(a) and 11(b) present the same results, but for a time interval later in the trace: $t \in[2000,2200]$. In this later interval, the number of vehicles is $20 \%$ higher than the previous two intervals. We observe in Fig. 11(a) that the match with the model is not good. Nevertheless, the curve formed by the points is almost linear, which indicates that the distribution is still Frechet. Fig. 11(b) confirms these conclusions. We also remark, as expected, that the model is optimistic compared to the data, because the data is obtained in a denser and thus more loaded network.

In this section, we observe that the model can accurately represent new data for the same network conditions. We also confirm a limitation of the EVT models: they are relevant only for a particular network situation. We can thus ask how we can deal with inhomogeneous networks? As we focus on extreme delay deviations, a good practice would be to be interested in the more conservative model (in our case the one obtained from the densest parts of the network).

\subsection{Discussions on the use and interpretation of EVT results}

EVT provides powerful tools to study large delays in a realistic situation. It allows the system designer to check if the actual system (or a very realistic simulation model) complies with the application requirements. EVT can also be seen as a complementary approach to formal methods: in the case a worst case delay has been derived using formal methods, it can be checked that the EVT probabilistic worst case is compatible with the one provided by formal methods. Moreover, during the system design process, several design options (different protocols, different protocol tunings, etc.) can be tested and their probabilistic worst case delays compared (by comparing the CDF of the obtained distributions) in order to choose the most suited for the considered application.

As mentioned previously, both BM and POT methods can be applied to the study of large delays in wireless networks, but they do not have the same signification. BM provides the probabilistic worst case delay, whereas POT provides a probability distribution of the delay knowing that it is already above a threshold. In the latter case, the probability of the delay to be 
above the threshold has to be known in order to provide the probabilistic bound on the delays in the network. As stated in section 3, POT provides $P(X-u<y \mid X>u)$, thus knowing $P(X>u)$, and thanks to the conditional probability formula we can compute $P(u<X<u+y)$. Nevertheless, $P(u<X<u+y)$ is not exactly equivalent to the result provided by the BM method: BM is about the maximum delay (the worst case), whereas POT is about all delays (including the maximum).

In sections 5.3 and 5.4 we investigate the impact of the size of the block and the value of the threshold on the EVT results. We show that if these values are not chosen high enough, the obtained delay distribution can be overly optimistic. A too optimistic bound can lead to a system not sufficiently safe. The size of the block and value of the threshold have thus to be chosen carefully: high enough so the fit is good (as in Figures 7(b) and 9(b)), but not too high so there is sufficient data to perform the fitting and the statistical test.

We can also remark that EVT can provide a probability of extreme deviation from the mean even if those deviations are never observed in reality. Nevertheless, the probability itself depends on the measurements performed on the observed system. If those measurements are not representative of the complete behavior of the system, the obtained probabilistic bound can be too optimistic [38]. The experimentation or simulation conditions have thus to be carefully chosen [38].

\section{Conclusion and future works}

This paper aims at studying large delays in wireless networks using the EVT statistical method. We first discuss the application of EVT to the study of large delays in networks. We then apply the two techniques provided by EVT: BM and POT, to the study of inter-beacon delays in VANETs safety applications. We observe that in the BM case, it is the Frechet distribution family which fits best the distribution of maximum inter-beacon delays. This distribution could thus be used as a model of probabilistic worst case in this context. We also remark that EVT cannot be applied in the case the measured delays are not identically distributed (as for the first considered data set). We study the impact of the block size and the threshold value (resp. for BM and POT method) and observe that to chose too low values can lead to a too optimistic probabilistic worst case delay and thus to overestimate the safety of the system. We conclude that EVT is a powerful tool which allows 
to estimate large delays in networks. It can be used as a complementary tool to formal methods when they fail to provide results or as a way to double check their results with more realistic system models.

EVT allows to deduce a model of the worst case delay based on data samples retrieve from simulation or experimentation. The sampling method is thus of uttermost importance: the sampled executions of the system must be representative of the whole set of its possible behaviors. If it is not the case, the EVT results might be biased. In the future, we plan to investigate the impact of the sampling methods on EVT results.

[1] H. Hartenstein, K. Laberteaux, VANET: vehicular applications and inter-networking technologies, Wiley Online Library, 2010.

[2] R. Jain, The art of computer systems performance analysis, John Wiley \& Sons, 2008.

[3] C. Baier, J.-P. Katoen, Principles of Model Checking, The MIT Press, 2008.

[4] J.-Y. Le Boudec, P. Thiran, Network calculus: a theory of deterministic queuing systems for the internet, Vol. LNCS 2050, Springer, 2001.

[5] Y. Lu, T. Nolte, I. Bate, L. Cucu-Grosjean, A statistical response-time analysis of real-time embedded systems., IEEE RTSS, San Juan, Puerto Rico, 2012, pp. 351-362.

[6] J. Beirlant, Y. Goegebeur, J. Segers, J. Teugels, Statistics of extremes: theory and applications, John Wiley \& Sons, 2006.

[7] S. Uppoor, M. Fiore, Large-scale urban vehicular mobility for networking research, IEEE VNC, Amsterdam, The Netherlands, 2011, pp. 6269 .

[8] H. Noori, B. Olyaei, A novel study on beaconing for vanet-based vehicle to vehicle communication: Probability of beacon delivery in realistic large-scale urban area using 802.11p, IEEE SaCoNeT, Paris, France, 2013, pp. 1-6.

[9] D. Jiang, L. Delgrossi, Ieee 802.11 p: Towards an international standard for wireless access in vehicular environments, IEEE VTC spring, Marina Bay, Singapore, 2008, pp. 2036-2040. 
[10] A. Mouradian, Study of probabilistic worst case inter-beacon delays under realistic vehicular mobility conditions, ADHOC-NOW 2015, Athens, Greece, 2014, pp. 390-403.

[11] E.-R. Olderog, H. Dierks, Real-time systems: formal specification and automatic verification, Cambridge University Press, 2008.

[12] E. Clarke, J. Wing, Formal methods: State of the art and future directions, ACM Computing Surveys 28 (4) (1996) 626-643.

[13] J. Baeten, A brief history of process algebra, Theoretical Computer Science 335 (2-3) (2005) 131-146.

[14] A. Fehnker, L. Van Hoesel, A. Mader, Modelling and verification of the lmac protocol for wireless sensor networks, IFM, Oxford, UK, 2007, pp. 253-272.

[15] M. Kwiatkowska, G. Norman, J. Sproston, Probabilistic model checking of the IEEE 802.11 wireless local area network protocol, Process Algebra and Probabilistic Methods: Performance Modeling and Verification (2002) 1-8.

[16] M. Kwiatkowska, G. Norman, R. Segala, J. Sproston, Automatic verification of real-time systems with discrete probability distributions, Theoretical Computer Science 282 (1) (2002) 101-150.

[17] M. Vojnovic, J.-Y. Le Boudec, Stochastic analysis of some expedited forwarding networks, IEEE INFOCOM, New York, USA, 2002, pp. 10041013.

[18] Y. Jiang, A basic stochastic network calculus, ACM SIGCOMM, Pisa, Italy, 2006, pp. 123-134.

[19] S. Edgar, A. Burns, Statistical analysis of wcet for scheduling, IEEE RTSS, London, UK, 2001, pp. 215-224.

[20] R. K. Shukla, M. Trivedi, M. Kumar, On the proficient use of gev distribution: a case study of subtropical monsoon region in india, Annals. Computer Science Series 8.

[21] E. Alvarado, D. V. Sandberg, S. G. Pickford, Modeling large forest fires as extreme events, National Emergency Training Center, 1998. 
[22] P. Embrechts, S. I. Resnick, G. Samorodnitsky, Extreme value theory as a risk management tool, North American Actuarial Journal 3 (2) (1999) 30-41.

[23] C. Liu, Y. Shu, J. Liu, O. Yang, Application of extreme value theory to the analysis of wireless network traffic, IEEE ICC, Glasgow, Scotland, 2007, pp. 486-491.

[24] M. Uchida, Traffic data analysis based on extreme value theory and its applications, IEEE GLOBECOM, Dallas, USA, 2004, pp. 1418-1424.

[25] A. Y. Dahab, A. bin Md Said, H. bin Hasbullah, Application of extreme value theory to bursts prediction, Signal Processing: An International Journal (SPIJ) 3 (4) (2009) 55.

[26] P. Liu, R. Berry, M. L. Honig, Delay-sensitive packet scheduling in wireless networks, IEEE WCNC, New Orleans, USA, 2003, pp. 1627-1632.

[27] J. A. Nelder, R. Mead, A simplex method for function minimization, The computer journal 7 (4) (1965) 308-313.

[28] scipy.optimize package, http://docs.scipy.org/doc/scipy-0.14.0/ reference/optimize.html, [Online; accessed 3-November-2014].

[29] scipy.stats package, http://docs.scipy.org/doc/scipy-0.14.0/ reference/stats.html, [Online; accessed 3-November-2014].

[30] C. V. S. C. Consortium, et al., Vehicle safety communications project: task 3 final report: identify intelligent vehicle safety applications enabled by dsrc, National Highway Traffic Safety Administration, US Department of Transportation, Washington DC.

[31] R. Stanica, E. Chaput, A.-L. Beylot, Broadcast communication in vehicular ad-hoc network safety applications, IEEE CCNC, Lax Vegas, USA, 2011, pp. 462-466.

[32] E. E. ETSI, 302665 V1. 1.1: Intelligent Transport Systems (ITS), Communications Architecture, European Standard (Telecommunications Series). 
[33] IEEE, Standard for Information Technology - Telecommunications and Information Exchange between Systems - Local and Metropolitan Area Networks - Specific Requirements - Part 11: Wireless LAN Medium Access Control (MAC) and Physical Layer (PHY) Specifications - Amendment 6: Wireless Access in Vehicular Environments (2010).

[34] B. Kloiber, C. Garcia, J. Härri, T. Strang, Update delay: A new information-centric metric for a combined communication and application level reliability evaluation of cam based safety applications, in: ITS World Congress, 2012.

[35] A. Autolitano, C. Campolo, A. Molinaro, R. M. Scopigno, A. Vesco, An insight into Decentralized Congestion Control techniques for VANETs from ETSI TS 102687 V1. 1.1, IFIP WD, Valencia, Spain, 2013, pp. $1-6$.

[36] The Network Simulator - ns-2, http://www.isi.edu/nsnam/ns/, [Online; accessed 5-November-2014].

[37] J. Scott Long, Regression models for categorical and limited dependent variables, Advanced quantitative techniques in the social sciences 7.

[38] J. Abella, E. Quinones, F. Wartel, T. Vardanega, F. J. Cazorla, Heart of gold: Making the improbable happen to increase confidence in mbpta, IEEE ECRTS, Madrid, Spain, 2014, pp. 255-265. 